Journal of the Brazilian Society of Mechanical Sciences and Engineering

PUBLISHER: SPRINGER; IMPACT FACTOR: 1.743

ISSN: 1678-5878 (PRINT VERSION); ISSN: 1806-3691 (ELECTRONIC VERSION)

Accepted August $31^{\text {st }} 2019$

\title{
BIOLOGICAL INTERACTIONS BETWEEN CARREAU FLUID AND MICRO- SWIMMERS IN A COMPLEX WAVY CANAL WITH MHD EFFECTS
}

\author{
N. Ali ${ }^{\mathrm{a}}$, Z. Asghar ${ }^{\mathrm{b}, *}$, M. Sajid ${ }^{\mathrm{a}}$ and O. Anwar Bégc \\ ${ }^{b}$ Department of Mathematics and Statistics, International Islamic University, Islamabad 44000, Pakistan \\ $b^{*}$ NUTECH School of Applied Sciences and Humanities, National University of Technology, Islamabad, 44000, Pakistan \\ ${ }^{c}$ Fluid Mechanics and Propulsion, Aeronautical and Mechanical Engineering Department, School of Science, Engineering and \\ Environment (SEE), Newton Building, University of Salford, M54WT, UK
}

\begin{abstract}
:
The efficient magnetic swimming of actual or mechanically designed micro-swimmers within bounded regions is reliant on several factors. Few of which are: the actuation of these swimmers via magnetic field, rheology of surrounding liquid (with dominant viscous forces), nature of medium (either porous or non-porous), position (either straight, inclined or declined) and state (either active or passive) of the narrow passage. To witness these interactions, we utilize Carreau fluid with Taylor swimming sheet model under magnetic and porous effects. Moreover, the cervical canal is approximated as a two-dimensional complex wavy channel inclined at certain angle with the horizontal. The momentum equations are reduced by means of lubrication assumption, which finally leads to a fourth order differential equation. MATLAB's built-in bvp4c function is employed to solve the resulting boundary value problem (BVP). The solution obtained via bvp4c is further verified by finite difference method (FDM). In both these methods, the refined values of flow rate and cell speed are computed by utilizing modified Newton-Raphson method. These realistic pairs are further utilized, to calculate the energy delivered by the micro-swimmer. The numerical results are plotted and discussed at the end of the article. Our study explains that the optimum speed of the micro-organism can be achieved by means of exploiting the fluid rheology and with the suitable application of the magnetic field. The peristaltic nature of the channel walls and porous medium may also serve as alternative factors to control the speed of the propeller.
\end{abstract}

Keywords: Magnetic field, complex wavy channel, porous medium, Carreau fluid, microswimmer, inclined channel.

*Corresponding author. Tel.: + 923455882685; E-mail address: zee.qau5@gmail.com 


\section{Nomenclature}

\section{Roman symbols}

$a_{S} \quad$ Wave amplitude in organism surface

$a_{1}, a_{2} \quad$ Wave amplitude in channel walls
C
Wave speed

$U_{S} \quad$ Swimming speed of the organism

$X, Y \quad$ Cartesian coordinates for fixed frame

$x, y \quad$ Cartesian coordinates for wave frame

Superscript (+) Upper half $\left(H_{S} \leq Y \leq H_{1}\right)$

Superscript (-) Lower half $\left(H_{2} \leq Y \leq H_{S}\right)$

V Velocity vector of the fluid

$V_{1}, V_{2} \quad$ Velocity components in fixed frame

$v_{1}, v_{2} \quad$ Velocity components in wave frame

$B_{0} \quad$ Strength of magnetic field

$\mathbf{F}_{\mathbf{C}} \quad$ Resultant force on the organism

$P \quad$ Pressure in fixed frame

p Pressure in wave frame

S Extra stress tensor

$\mathbf{A}_{1} \quad$ First Rivlin-Ericksen tensor

$S_{x x}, S_{x y}$ and $S_{y y} \quad$ Components of extra

\section{Greek symbols}

$\lambda$

Wavelength

$\Phi \quad$ Phase difference $(0 \leq \phi \leq \pi)$

$\rho$

K

$\sigma_{m}$

$\alpha$

$\mu_{0} \quad$ Zero shear-rate viscosity

$\mu_{\infty} \quad$ Infinite shear-rate viscosity

$\gamma \quad$ Ratio of infinite to zero shearrate viscosity

Second invariant

$\Gamma$

$\delta$

Time constant/ relaxation time

Dimensionless wave number

$\Psi$

Stream function

\&o Power delivered by the swimmer

$\xi_{j} \quad$ Unit vector normal to the organism

$\Omega \quad$ Under-relaxation parameter $\Omega \in(0,1]$ 


$\begin{array}{ll}\text { We } & \text { Weissenberg number } \\ n & \text { Power law index } \\ R e & \text { Reynolds number } \\ H & \text { Hartmann number } \\ g & \text { Force due to gravity } \\ D_{a} & \text { Darcy number } \\ G & \text { Gravitational parameter } \\ F & \text { Flow rate of the fluid }\end{array}$

\section{INTRODUCTION}

An undulating sheet or an undulating cylindrical filament in a viscous fluid serve as a mathematical model for swimming of a minute organism. Both these models are thoroughly analyzed by Taylor in two different communications $[1,2]$. These pioneer studies have laid down foundation for most of the later contributions in the field of swimming microorganisms. Taylor utilized lubrication theory along with perturbation method to compute the speed and efficiency of the organism. An alternative approach based on stokeslet distribution technique was employed by Hancock [3] to calculate the speed of a cylindrical filament in a Newtonian viscous fluid. Gray and Hancock [4] successfully computed the speed of a sea urchin spermatozoa by employing the general theory of flagella motion. Extension of Taylor's work to account for large amplitude undulations in the tail of the microorganism was made by Drummond [5]. The inclusion of the inertial part of the momentum equation in the Taylor analysis was substantiated by Reynolds [6] and Tuck [7]. The motion of microorganism next to a solid boundary or inside a tubular structure is naturally justified to be analyzed because of its relevance with actual environment surrounding the microorganism such as spermatozoon. With such motivation, Reynolds [6] modeled the motion of microorganism near a solid wall and predicted a repulsive motion of the organism from the wall. The dynamics of microorganism between two rigid passive walls based on the lubrication theory was investigated by Shack and Lardner [8]. The effects of a thin peripheral layer fluid surrounding a thick core 
layer fluid of different viscosity on swimming of a minute organism through a channel were thoroughly discussed by Shukla et al. [9]. The observation that the spermatozoon motion is supplemented with the contraction of the cervix and uterus during its journey towards oviducts has led to the study performed by Smelser et al. [10]. In this study, the authors developed a mathematical model of sperm movement through a channel with wavy walls. The model of Smelser et al. [10] was studied under long wavelength theory by Shukla et al. [11]. Radhakrishnamacharya and Sharma [12] quantified the role of variable viscosity on the movement of spermatozoa in a passive rigid channel.

The rheology of fluid in all the above mentioned studies is not treated as a crucial factor in order to achieve the efficient swimming despite the fact that cervical fluid exhibits remarkable nonNewtonian characteristics. This fact motivated several researchers to explore the dynamics of swimming of microorganism through a complex rheological fluid. A brief overview of these attempts is as follows: Tuck's works on inertial swimming was extended by Chaudhry [13] for viscoelastic second order fluid. His analysis revealed a reduction in the swimming speed with increasing the fluid elasticity at larger Reynolds number. However this behavior was due to a calculation error which was pointed out by Sajid et al. [14] in their study on magnetohydrodynamics effects in inertial swimming through an infinite pool of second order fluid. Sturges [15] employed a complex integral model to study the dynamics of swimming microorganism in an infinite quiescent fluid. The explicit formulas of swimming speed and energy consumed by the organism for several non-Newtonian models were derived by Lauga [16]. He emphasized on the role of non-Newtonian characteristics of the fluid to optimize the speed and energy expended by the organism. Lauga's work was extended by Sajid $\boldsymbol{e t}$ al. [17] to account for the porosity effects. Ali et al. [18] used couple stress model to quantify the rotational effects of the fluid particles on the swimming characteristics in an unbounded medium. The celebrated Eringen micropolar model was utilized by Sinha et al. [19] and Phillip and Chandra [20] to estimate the micro-rotational effects on swimming hydrodynamics. The Bingham model was integrated by Balmforth et al. [21] to study the swimming of microorganism near to a rigid wall. More recently, Ives and Morozov [22] presented an analysis of swimming in a viscoelastic Oldroyd-B fluid in the presence of a solid wall. The computations were carried out by an efficient spectral method. Their results indicate that viscoelasticity can enhance the speed of the microorganism in the presence of a rigid boundary. 
The interaction of magnetic field with swimming dynamics of microorganism is important due to its applications in bio-medical industry. These include but not limited to micro-magneto-robots used in non-invasive medical procedures [23], treatment of diseased eyes through targeted drug delivery [24], evaluating the robustness of prosthetic smart magneto rheological kneecaps [25] and green magneto-tactic fuel cells $[\mathbf{2 6}, \mathbf{2 7}]$. The previous study on swimming of microorganism in a magneto-hydrodynamic (MHD) environment were carried out by Ansari et al. [28] for swimming of microorganism in a second grade fluid, Gadelha [29] on swimming in the presence of oscillating magnetic fluid, Belovs and Cebers [30] for ferromagnetic swimmers, Goa et al. [31] on application of free magnetic swimming to targeted drug deliver, Gauger $\boldsymbol{e t}$ al. [32] on the role magnetically actuated cilia in transportation of fluid at low Reynolds number and Asghar $\boldsymbol{e t}$ al. [33] on hydromagnetic swimming of Carreau fluid in an active channel.

Motivated by above studies the aim of this paper is to explore the swimming characteristics in a magneto-hydrodynamic Carreau fluid by including three novel effects which are: (i) the passive transport mechanism effects achieved through peristaltic activity governed by complex waves (ii) the channel inclination effects (iii) the permeability effects. The previous study pertaining to the effects of porous medium on swimming in an unbounded medium were carried out by Sajid $\boldsymbol{e t}$ al. [17] and Ansari et al. [34]. The peristaltic motion included in the present model greatly resembles with the cilia motion as both these mechanisms contribute toward biological fluid transport processes. We refer the readers to studies carried out in refs. [35-41] for details on theoretical models pertaining to the cilia assisted fluid transport. The Carreau fluid model chosen for the analysis is elegant because of its superiority over other generalized Newtonian fluid models in predicting the rheology of many biological fluids. This superiority of Carreau model owes to five parameters which appear in its constitutive equation and which provide it a great flexibility to fit the viscosity shear rate of several physiological and industrial fluids [42]. In context of swimming microorganism, the Carreau model has been employed by Cordero and Lauga [43] to generalize the Taylor undulating sheet model for complex rheological fluids. The application of Carreau model in related areas such as gliding motion of bacteria, swimming of spermatozoa in passive channel and peristaltic flows are documented in refs. [44-46].

The present work is structured in the following sequence: In section 2, geometrical description of the problem and extra stress tensor of Carreau model are presented. Problem is modeled in section 
3. Solution methodology is elaborated in section 4. Validation of the obtained solution with FDM is explained in section 5. The effects of several parameters on cell speed, flow rate and power dissipation are displayed in $\boldsymbol{M A T L A B}$ figures and explained in section 6. Lastly, section 7 concludes the whole work.

\section{PHYSICAL DIAGRAM AND EQUATIONS OF CARREAU MODEL}

Fig. 1 presents a magnetically controlled undulating sheet swimming through a Carreau fluid bounded between two inclined active walls. We also consider the porous medium effects in the present work. Due to its wavy surface, the micro-swimmer is propelling in the inclined negative $X$-direction. A magnetic field of constant strength (applied in the negative $Y$-direction) is also superimposed on the swimming hydrodynamics.

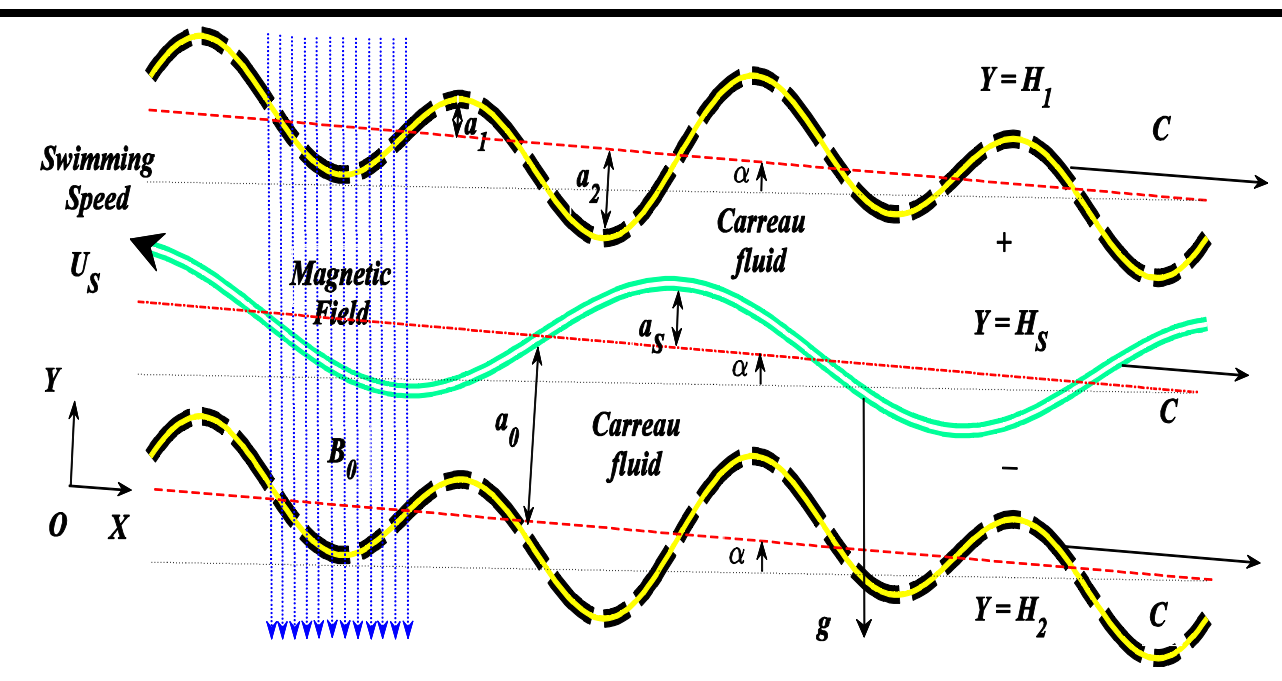

Fig.1. Propulsion of magnetic micro-swimmer through complex wavy inclined channel

In the fixed frame of reference, the complex waves at upper $\left(Y=H_{1}\right)$ and lower walls $\left(Y=H_{2}\right)$ and the undulating surface $\left(Y=H_{S}\right)$ of the organism are mathematically defined as:

$$
\begin{gathered}
H_{1}=a_{0}+a_{1} \sin \left[\frac{2 \pi}{\lambda}\left(X-\left(C-U_{S}\right) t\right)\right]+a_{2} \sin \left[\frac{4 \pi}{\lambda}\left(X-\left(C-U_{S}\right) t\right)\right] \\
H_{S}=a_{S} \sin \left[\frac{2 \pi}{\lambda}\left(X-\left(C-U_{S}\right) t\right)+\Phi\right] \\
H_{2}=-a_{0}+a_{1} \sin \left[\frac{2 \pi}{\lambda}\left(X-\left(C-U_{S}\right) t\right)\right]+a_{2} \sin \left[\frac{4 \pi}{\lambda}\left(X-\left(C-U_{S}\right) t\right)\right],
\end{gathered}
$$


Here $C-U_{S}$ is basically the speed of the wave relative to the organism.

The fluid velocity components at the boundary walls are constrained according to the following conditions.

$$
\begin{aligned}
& \left.V_{1}^{+}=0\right\} \quad \text { at } Y=H_{1} \\
& \left.V_{2}^{-}=0\right\} \text { and } Y=H_{2}{ }^{\prime} \\
& \left.\begin{array}{c}
V_{1}^{ \pm}=-U_{S} \\
V_{2}^{ \pm}=-\frac{d H_{S}}{d t}
\end{array}\right\} \text { at } Y=H_{S} .
\end{aligned}
$$

For free swimmer case, the net force acting on the organism must vanish. This condition also referred as the dynamic equilibrium condition may be casted mathematically as $[\mathbf{3 3}, \mathbf{3 7}, \mathbf{4 1}]$ :

$$
\int\left(\mathbf{F}_{\mathbf{C}}^{+}+\mathbf{F}_{\mathbf{C}}^{-}\right) d s=0
$$

The relevant equations governing the flow are:

$$
\begin{gathered}
\nabla \cdot \mathbf{V}^{ \pm}=0 . \\
\rho \frac{d \mathbf{V}^{ \pm}}{d t}=-\nabla p^{ \pm}+\nabla \cdot \mathbf{S}^{ \pm}+\rho \mathbf{B}^{ \pm} .
\end{gathered}
$$

The extra stress tensor of Carreau model is [39-42]:

$$
\mathbf{S}^{ \pm}=\left(\mu_{\infty}+\left(\mu_{0}-\mu_{\infty}\right)\left(1+\Gamma^{2}\left(\Pi^{ \pm}\right)^{2}\right)^{\frac{n-1}{2}}\right) \mathbf{A}_{1}^{ \pm}
$$

where $\Pi^{ \pm}$is given as:

$$
\Pi^{ \pm}=\sqrt{\frac{1}{2}\left(\mathbf{A}_{1}^{ \pm}: \mathbf{A}_{1}^{ \pm}\right)}=\sqrt{\frac{1}{2} \operatorname{trace}\left(\left(\mathbf{A}_{1}^{ \pm}\right)^{2}\right)}, \quad \mathbf{A}_{1}^{ \pm}=\left(\nabla \mathbf{V}^{ \pm}\right)+\left(\nabla \mathbf{V}^{ \pm}\right)^{T}
$$

The Galilean transformations connecting variables in fixed and wave frames are:

$$
\begin{gathered}
v_{1}^{ \pm}=V_{1}^{ \pm}-\left(C-U_{S}\right), \quad v_{2}^{ \pm}=V_{2}^{ \pm}, \quad P^{ \pm}=p^{ \pm}, \\
x=X-\left(C-U_{S}\right) t, \quad y=Y .
\end{gathered}
$$




\section{MAGNETIC SWIMMING MODEL WITH POROUS MEDIUM}

The appropriate velocity field for the flow induced by combined motion of the organism and the wavy walls is defined as $\mathbf{V}^{ \pm}=\left[v_{1}^{ \pm}, v_{2}^{ \pm}, 0\right]$. Further, the body force arising due to combination of gravity, magnetic field and porous medium is given by $\mathbf{B}=\sigma B_{0}^{2}$. For this velocity, Eqs. (7) and (8) become:

$$
\begin{gathered}
\frac{\partial v_{1}^{ \pm}}{\partial x}+\frac{\partial v_{2}^{ \pm}}{\partial y}=0 \\
\rho\left(v_{1}^{ \pm} \frac{\partial}{\partial x}+v_{2}^{ \pm} \frac{\partial}{\partial y}\right) v_{1}^{ \pm}=-\frac{\partial p^{ \pm}}{\partial x}+\frac{\partial S_{x x}^{ \pm}}{\partial x}+\frac{\partial S_{x y}^{ \pm}}{\partial y}+\left(\sigma_{m} B_{o}^{2}-\frac{\mu_{0}}{K}\right) v_{1}^{ \pm}+\rho g \sin \alpha \\
\rho\left(v_{1}^{ \pm} \frac{\partial}{\partial x}+v_{2}^{ \pm} \frac{\partial}{\partial y}\right) v_{2}^{ \pm}=-\frac{\partial p^{ \pm}}{\partial y}+\frac{\partial S_{x y}^{ \pm}}{\partial x}+\frac{\partial S_{y y}^{ \pm}}{\partial y}-\frac{\mu_{0}}{K} v_{2}^{ \pm}-\rho g \cos \alpha \\
S_{x x}^{ \pm}=2\left(\mu_{\infty}+\left(\mu_{0}-\mu_{\infty}\right)\left(1+\Gamma^{2}\left(\Pi^{ \pm}\right)^{2}\right)^{\frac{n-1}{2}}\right)\left(\frac{\partial v_{1}^{ \pm}}{\partial x}\right) \\
S_{x y}^{ \pm}=\left(\mu_{\infty}+\left(\mu_{0}-\mu_{\infty}\right)\left(1+\Gamma^{2}\left(\Pi^{ \pm}\right)^{2}\right)^{\frac{n-1}{2}}\right)\left(\frac{\partial v_{1}^{ \pm}}{\partial y}+\frac{\partial v_{2}^{ \pm}}{\partial x}\right) \\
S_{y y}^{ \pm}=2\left(\mu_{\infty}+\left(\mu_{0}-\mu_{\infty}\right)\left(1+\Gamma^{2}\left(\Pi^{ \pm}\right)^{2}\right)^{\frac{n-1}{2}}\right)\left(\frac{\partial v_{2}^{ \pm}}{\partial y}\right)
\end{gathered}
$$

where

$$
\left(\Pi^{ \pm}\right)^{2}=\left(\left(\frac{\partial v_{1}^{ \pm}}{\partial x}\right)^{2}+\left(\frac{\partial v_{2}^{ \pm}}{\partial y}\right)^{2}\right)+\frac{1}{2}\left(\frac{\partial v_{1}^{ \pm}}{\partial y}+\frac{\partial v_{2}^{ \pm}}{\partial x}\right)^{2}
$$

Introducing: 


$$
\begin{gathered}
\left(\Pi^{ \pm}\right)^{2^{*}}=\frac{a_{0}}{C}\left(\Pi^{ \pm}\right)^{2}, a_{1,2, S}{ }^{*}=\frac{a_{1,2, S}}{a_{0}}, U_{S}^{*}=\frac{U_{S}}{C}, \mathcal{P}^{*}=\frac{2 \pi a_{0}}{\lambda \mu_{0} C^{2}} \mathcal{P}, G=\frac{\rho g a_{0}^{2}}{\mu_{0} C} \\
H_{1,2, S}{ }^{*}=\frac{H_{1,2, S}}{a_{0}}, \delta=\frac{2 \pi a_{0}}{\lambda}, R e=\frac{\rho C a_{0}}{\mu_{0}}, W e=\frac{\Gamma C}{a_{0}}, \gamma=\frac{\mu_{\infty}}{\mu_{0}}, H^{2}=\frac{\sigma_{m} a_{0}^{2}}{\mu_{0}} B_{o}^{2}, D_{a}=\frac{K}{a_{0}^{2}} \\
x^{*}=\frac{2 \pi}{\lambda} x, y^{*}=\frac{y}{a_{0}},\left(v_{1}^{ \pm}\right)^{*}=\frac{v_{1}^{ \pm}}{C},\left(v_{2}^{ \pm}\right)^{*}=\frac{v_{2}^{ \pm}}{\delta C},\left(p^{ \pm}\right)^{*}=\frac{2 \pi a_{0}^{2}}{\lambda \mu_{0} C} p^{ \pm},\left(S^{ \pm}{ }_{i j}\right)^{*}=\frac{a_{0}}{\mu_{0} C} S^{ \pm}{ }_{i j},
\end{gathered}
$$

Defining:

$$
\left(v_{1}^{ \pm}\right)^{*}=\frac{\partial \Psi^{ \pm}}{\partial y^{*}}, \quad\left(v_{2}^{ \pm}\right)^{*}=-\frac{\partial \Psi^{ \pm}}{\partial x^{*}}
$$

Utilizing (19) and (20) in (13) - (18) and neglecting superscript*, we get:

$$
\begin{gathered}
\delta \operatorname{Re}\left(\left(\frac{\partial \Psi^{ \pm}}{\partial y} \frac{\partial}{\partial x}-\frac{\partial \Psi^{ \pm}}{\partial x} \frac{\partial}{\partial y}\right)\left(\frac{\partial \Psi^{ \pm}}{\partial y}\right)\right)= \\
\delta \frac{\partial}{\partial x} S_{x x}^{ \pm}+\frac{\partial}{\partial y} S_{x y}^{ \pm}-\frac{\partial p^{ \pm}}{\partial x}+\left(H^{2}-\frac{1}{D_{a}}\right) \frac{\partial \Psi^{ \pm}}{\partial y}+G \sin \alpha \\
-\delta^{3} \operatorname{Re}\left[\left(\frac{\partial \Psi^{ \pm}}{\partial y} \frac{\partial}{\partial x}-\frac{\partial \Psi^{ \pm}}{\partial x} \frac{\partial}{\partial y}\right)\left(\frac{\partial \Psi^{ \pm}}{\partial x}\right)\right]= \\
\delta^{2} \frac{\partial}{\partial x} S_{x y}^{ \pm}+\delta \frac{\partial}{\partial y} S^{ \pm}{ }_{y y}-\frac{\partial p^{ \pm}}{\partial y}-\frac{1}{D_{a}} \delta^{2} \frac{\partial \Psi^{ \pm}}{\partial x}-\delta G \cos \alpha \\
S_{x y}^{ \pm}=\left(\gamma+(1-\gamma)\left(1+W e^{2}\left(\Pi^{ \pm}\right)^{2}\right)^{\frac{n-1}{2}}\right)\left(\frac{\partial^{2} \Psi^{ \pm}}{\partial y^{2}}-\delta^{2} \frac{\partial^{2} \Psi^{ \pm}}{\partial x^{2}}\right) \\
S_{y y}^{ \pm}=2 \delta\left(\gamma+(1-\gamma)\left(1+W e^{2}\left(\Pi^{ \pm}\right)^{2}\right)\left(1+W e^{2}\left(\Pi^{ \pm}\right)^{2}\right)^{\frac{n-1}{2}}\right)\left(\frac{\partial^{2} \Psi^{ \pm}}{\partial x \partial y}\right) \\
\left(\frac{\partial^{2} \Psi^{ \pm}}{\partial x \partial y}\right)
\end{gathered}
$$

With non-dimensional second invariant:

$$
\left(\Pi^{ \pm}\right)^{2}=2\left(\delta \frac{\partial^{2} \Psi^{ \pm}}{\partial x \partial y}\right)^{2}+\frac{1}{2}\left(\frac{\partial^{2} \Psi^{ \pm}}{\partial y^{2}}-\delta^{2} \frac{\partial^{2} \Psi^{ \pm}}{\partial x^{2}}\right)^{2}
$$


Employing creeping flow and long wavelength assumption [1-9, 11, 12, 33-38, 40-49] to Eqn. (23)-(26), we have $S_{y y}^{ \pm}=S_{x x}^{ \pm}=0, S_{x y}^{ \pm}=\left(\gamma+(1-\gamma)\left(1+W e^{2}\left(\Pi^{ \pm}\right)^{2}\right)^{\frac{n-1}{2}}\right)\left(\frac{\partial^{2} \Psi^{ \pm}}{\partial y^{2}}\right) \quad$ and $\left(\Pi^{ \pm}\right)^{2}=\frac{1}{2}\left(\frac{\partial^{2} \Psi^{ \pm}}{\partial y^{2}}\right)^{2}$

Combining these compact expressions into reduced Eqn. (21), we get:

$$
\frac{\partial p^{ \pm}}{\partial x}=\frac{\partial}{\partial y}\left\{\left(\gamma+(1-\gamma)\left(1+W e^{2}\left(\Pi^{ \pm}\right)^{2}\right)^{\frac{n-1}{2}}\right)\left(\frac{\partial^{2} \Psi^{ \pm}}{\partial y^{2}}\right)\right\}+\left(H^{2}-\frac{1}{D_{a}}\right) \frac{\partial \Psi^{ \pm}}{\partial y}+G \sin \alpha
$$

The compatibility equation is:

$$
\frac{\partial^{2}}{\partial y^{2}}\left\{\left(\gamma+(1-\gamma)\left(1+W e^{2}\left(\Pi^{ \pm}\right)^{2}\right)^{\frac{n-1}{2}}\right)\left(\frac{\partial^{2} \Psi^{ \pm}}{\partial y^{2}}\right)\right\}+\left(H^{2}-\frac{1}{D_{a}}\right) \frac{\partial^{2} \Psi^{ \pm}}{\partial y^{2}}=0
$$

After utilizing the Galilean transformation, dimensionless variables and stream function on Eqn. (4) and (5), we finally arrive at the boundary conditions:

$$
\begin{aligned}
& \left.\Psi^{ \pm}=0, \quad \frac{\partial \Psi^{ \pm}}{\partial y}=U_{S}-1\right\} \text { at } y=H_{1}=1+a_{1} \sin x+a_{2} \sin 2 x \\
& \frac{\partial \Psi^{ \pm}}{\partial x}=0 \quad \quad \text { and } y=H_{2}=-1+a_{1} \sin x+a_{2} \sin 2 x, \\
& \left.\begin{array}{c}
\Psi^{ \pm}=\mp F, \quad \frac{\partial \Psi^{ \pm}}{\partial y}=-1 \\
\frac{\partial \Psi^{ \pm}}{\partial x}=-a_{S} \cos (x+\Phi)
\end{array}\right\} \text { at } y=H_{S}=a_{S} \sin (x+\Phi)
\end{aligned}
$$

The standard definition of flow rate across two halves of the cervical canal:

$$
F=\int_{y=H_{2}}^{y=H_{S}} \frac{\partial \Psi^{-}}{\partial y} d y=\int_{y=H_{S}}^{y=H_{2}} \frac{\partial \Psi^{+}}{\partial y} d y
$$

The appropriate form of force balance condition (6) via utilizing stress-strain relation and long wavelength approximation is as follows: 


$$
\int_{-\pi}^{\pi}\left[S_{x y}\left(U_{S}, F ; x\right)\right]_{y=H_{S}}-H_{S}\left[\frac{d p\left(U_{S}, F ; x\right)}{d x}\right] d x=0 .
$$

Where $\left[S_{x y}\left(U_{S}, F ; x\right)\right]_{y=H_{S}}=\left.S_{x y}^{+}\left(U_{S}, F ; x\right)\right|_{y=H_{S}}-\left.S_{x y}^{-}\left(U_{S}, F ; x\right)\right|_{y=H_{S}}$, and pressure gradient $\left[\frac{d p\left(U_{S}, F ; x\right)}{d x}\right]=\frac{d p^{+}\left(U_{S}, F ; x\right)}{d x}-\frac{d p^{-}\left(U_{S}, F ; x\right)}{d x}$.

For the case of free channel:

$$
\Delta P_{\lambda}=\int_{-\pi}^{\pi} \frac{d p^{ \pm}}{d x} d x=p^{ \pm}(\pi)-p^{ \pm}(-\pi)=0
$$

The power losses can be calculated as:

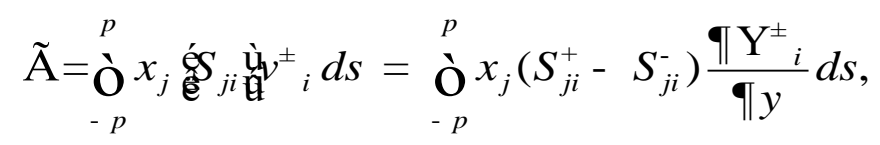

The final expression of power required (after using the lubrication assumption) is:

$$
\wp\left(U_{S}, F\right)=\int_{-\pi}^{\pi} \frac{d H_{S}}{d x}\left[p\left(U_{S}, F ; x\right)\right] d x=\int_{-\pi}^{\pi} a_{S} \cos (x+\Phi)\left(p^{+}-p^{-}\right) d x
$$

\section{SOLUTION METHODOLOGY}

In case of Newtonian mucus $(n=1$ or $W e=0)$, Eqn. (27) becomes:

$$
\frac{\partial^{3} \Psi^{ \pm}}{\partial y^{3}}+\left(H^{2}-\frac{1}{D_{a}}\right) \frac{\partial \Psi^{ \pm}}{\partial y}-\frac{\partial p^{ \pm}}{\partial x}+G \sin \alpha=0
$$

The closed form solution of (36) corresponding to the boundary conditions (29) and (30) is:

$$
\begin{aligned}
& \psi^{ \pm}= \frac{-\sqrt{D_{a}}}{\left(1-H^{2} D_{a}\right)^{3 / 2}}\left(y \sqrt{D_{a}} \sqrt{1-H^{2} D_{a}}\left(\frac{\partial p^{ \pm}}{\partial x}-G \sin \alpha\right)+\right. \\
&\left.\quad \operatorname{csch}\left(A_{1}^{ \pm}\right)\left(\cosh \left(A_{2}^{ \pm}\right)\left(\left(\frac{\partial p^{ \pm}}{\partial x}-G \sin \alpha+H^{2}\right) D_{a}-1\right)+A_{3}^{ \pm}\right)\right),
\end{aligned}
$$




$$
\begin{gathered}
\frac{d p^{ \pm}}{d x}=\frac{1}{D_{a}\left(\sqrt{1-H^{2} D_{a}}\left(H_{S}-H_{1,2}\right)+2 \sqrt{D_{a}} \tanh \left(A_{1}^{ \pm} / 2\right)\right)}\left(\sqrt { 1 - H ^ { 2 } D _ { a } } \left(F-D_{a}\left(F H^{2}-\right.\right.\right. \\
\left.\left.\left.G \sin \alpha\left(H_{S}-H_{1,2}\right)\right)\right)+\sqrt{D_{a}}\left(2+D_{a}\left(2 G \sin \alpha+H^{2}\left(U_{S}-2\right)\right)-U_{S}\right) \tanh \left(A_{1}^{ \pm} / 2\right)\right), \\
\text { where } A_{1}^{ \pm}=\frac{\sqrt{1-H^{2} D_{a}}\left(H_{1,2}-H_{S}\right)}{\sqrt{D_{a}}}, A_{2}^{ \pm}=\frac{\sqrt{1-H^{2} D_{a}}\left(H_{1,2}-y\right)}{\sqrt{D_{a}}}, \\
A_{3}^{ \pm}=\cosh \left(A_{2}^{ \pm}\right)\left(1+D_{a}\left(G \sin \alpha-\frac{\partial p^{ \pm}}{\partial x}+H^{2}\left(U_{S}-1\right)\right)-U_{S}\right) .
\end{gathered}
$$

For Newtonian fluid the equilibrium conditions to calculate the unknowns $F$ and $U_{S}$ are:

$$
\int_{-\pi}^{\pi} \frac{d p^{ \pm}}{d x} d x=0 \quad \text { and }\left.\int_{-\pi}^{\pi}\left(\frac{\partial^{2} \Psi^{+}}{\partial y^{2}}-\frac{\partial^{2} \Psi^{-}}{\partial y^{2}}\right)\right|_{y=H_{S}}-H_{S}\left(\frac{\partial p^{+}}{\partial x}-\frac{\partial p^{-}}{\partial x}\right) d x=0
$$

Utilizing Eqn. (37) and (38) into Eqn. (39) one can find out that the analytical integration technique is not possible due to nonlinear nature of these algebraic equations. Hence we need a numerical root finding algorithm to compute $F$ and $U_{S}$. We employed modified Newton-Raphson method in the present computations.

However, in the absence of magnetic field and without any porous medium effects one can obtain much simpler expressions of stream function and pressure gradient (which can be easily integrated as in Eqn. (39)). Finally, the solution of two algebraic equations with two unknowns is:

$$
\begin{aligned}
& F=\frac{1-\left(a_{1}-a_{S}\right)^{2}}{a_{1}^{2}+a_{1} a_{S}-2 a_{S}^{2}-1}-\frac{G \sin \alpha\left(1-\left(a_{1}-a_{S}\right)^{2}\right)^{3 / 2}}{12 \pi}, \\
& U_{S}=\frac{3\left(a_{1}^{2}-a_{S}^{2}\right)}{a_{1}^{2}+a_{1} a_{S}-2 a_{S}^{2}-1}-\frac{G \sin \alpha\left(1-\left(a_{1}-a_{S}\right)^{2}\right)^{1 / 2}}{4 \pi} .
\end{aligned}
$$

These expression of $F$ and $U_{S}$ are obtained in a special case of Newtonian mucus without porous/ magnetic effects. Flow rate and organism speed are functions of wave amplitude of channel and organism with inclination angle. In that case there is no alternative way to control the swimmer via magnetic field, permeability and rheological properties of surrounding fluids. However, (for non-Newtonian fluid with magnetic and porous effects) Eqns. (27) and (28) fulfill the purpose. 
Since these equations are highly non-linear so we need an appropriate numerical technique to solve the BVP (Eqns. (28), (29) and (30)). We solve the problem in MATLAB software via bvp4c solver. The solution is computed in two regions $H_{2} \leq y \leq H_{S}, H_{S} \leq y \leq H_{1}$ for $-\pi \leq x \leq \pi$ with some fixed values of $H, D_{a}, \gamma, W e, n, G, \alpha, \Phi, a_{1}, a_{2}$ and $a_{S}$. The two unknowns $F$ and $U_{S}$ are also assigned some initial values so that the BVP can be solved. The solution is further utilized in equilibrium conditions (which are off course not satisfied due to crude values of $F$ and $U_{S}$ ) to calculate the iterative values of flow rate and organism speed.

\section{PROBLEM VALIDATION WITH FDM}

The bvp4c solution is further validated by another numerical technique i.e. FDM. First we transform the nonlinear differential equation into linear one at $(j+1)^{\text {th }}$ step as:

$$
\begin{gathered}
\left(\frac{\partial^{2} q^{(j)}}{\partial y^{2}}+H^{2}-\frac{1}{D_{a}}\right) \frac{\partial^{2}\left(\Psi^{ \pm}\right)^{(j+1)}}{\partial y^{2}}+\frac{\partial q^{(j)}}{\partial y} \frac{\partial^{3}\left(\Psi^{ \pm}\right)^{(j+1)}}{\partial y^{3}}+q^{(j)} \frac{\partial^{4}\left(\Psi^{ \pm}\right)^{(j+1)}}{\partial y^{4}}=0 \\
\left(\Psi^{ \pm}\right)^{(j+1)}=0, \quad \frac{\partial\left(\Psi^{ \pm}\right)^{(j+1)}}{\partial y}=U_{S}-1 \text { at } y= \pm 1+a_{1} \sin x+a_{2} \sin 2 x, \\
\left(\psi^{ \pm}\right)^{(j+1)}=\mp F, \frac{\partial\left(\psi^{ \pm}\right)^{(j+1)}}{\partial y}=-1 \text { at } y=a_{S} \sin (x+\Phi),
\end{gathered}
$$

where

$$
q^{(j)}(y)=\gamma+(1-\gamma)\left(1+W e^{2}\left(\frac{\partial^{2}\left(\Psi^{ \pm}\right)^{(j)}}{\partial y^{2}}\right)^{2}\right)^{\frac{n-1}{2}}
$$

The above BVP is linear in $\left(\Psi^{ \pm}\right)^{(j+1)}$. Using central difference formulae, the BVP can be transformed into system of algebraic equations. This system can be solved (by matrix inverse method) to get $\left(\Psi^{ \pm}\right)^{(j+1)}$ at each cross-section. In order to get the convergent values of $\left(\Psi^{ \pm}\right)^{(j+1)}$ 
to $10^{-10}$ (which is denoted by $\left(\tilde{\Psi}^{ \pm}\right)^{(j+1)}$ ) we use successive under-relaxation (SUR) method which is:

$$
\left(\psi^{ \pm}\right)^{(k+1)}=\left(\psi^{ \pm}\right)^{(k)}+\Omega\left\{\left(\tilde{\psi}^{ \pm}\right)^{(k+1)}-\left(\psi^{ \pm}\right)^{(k)}\right\}
$$

The procedure of utilizing the FDM solution in equilibrium conditions to compute the $F$ and $U_{S}$ is exactly the same as that of bvp4c. The validation via FDM is shown by red dots Figs. 2 and 3.

\section{RESULTS AND DISCUSSION}

The numerical results obtain by means of bvp4c (validated by FDM) are shown through plots (in Figs. 2-9) and briefly discussed.

\subsection{FLOW RATE, CELL SPEED AND POWER DISSIPATION}

The role of magnetic field, fluid rheology and complex bounded domain in the swimming speed, flow rate and power dissipation is elaborated in Fig. 2. The speed of spermatozoa swimming through shear-thinning mucus against Weissenberg number is displayed in Fig. 2(a). Three curves are plotted for different magnetic strengths i.e. $H=0,0.25$ and 0.5. A single red colored curve is plotted for passive channel (without any wave at the channel walls $\left(a_{1}=a_{2}=0\right)$ ). This figure insures that the presence of magnetic field aids the micro-swimmer and a relatively greater speed is achieved for nonzero Hartmann number as compared to zero Hartmann number. The direction of the magnetic field is also an important aspect. If the direction become opposite (i.e. upward) the MHD will play a resistive role in organism swimming mechanism. In a similar fashion rheology of the surrounding fluid is also a key factor in faster or slower propulsion. For shear thinning case $(n<1)$ the organism attain a maximum speed for a small Weissenberg number and then decreases for large Weissenberg number just like a plateau-type topology. Moreover, Fig. 2(a) clearly reveals that the complex wavy channel appears to be a resistive factor since the speed of the organism is relatively greater in passive channel case.

The other unknown i.e. the flow rate of Carreau fluid is plotted in Fig. 2(b) for the same physical conditions as used in Fig. 2(a). This figure shows that the magnitude of flow rate reduces in the presence of magnetic field, while passive (rigid) canal increases the flow rate. In case of rigid 
channel there is more space available to the organism and it can propel easily (faster as compared to complex wavy channel) and pushes the fluid backward with greater speed.

These numerically calculated (and plotted) pairs of $F$ and $U_{S}$ are further utilized in Eqn. (35) to predict the behavior of power consumed by the micro-swimmer. Fig. 2(c) displaying the power dissipation is in good agreement with previous figures (Fig. 2(a) and (b)).
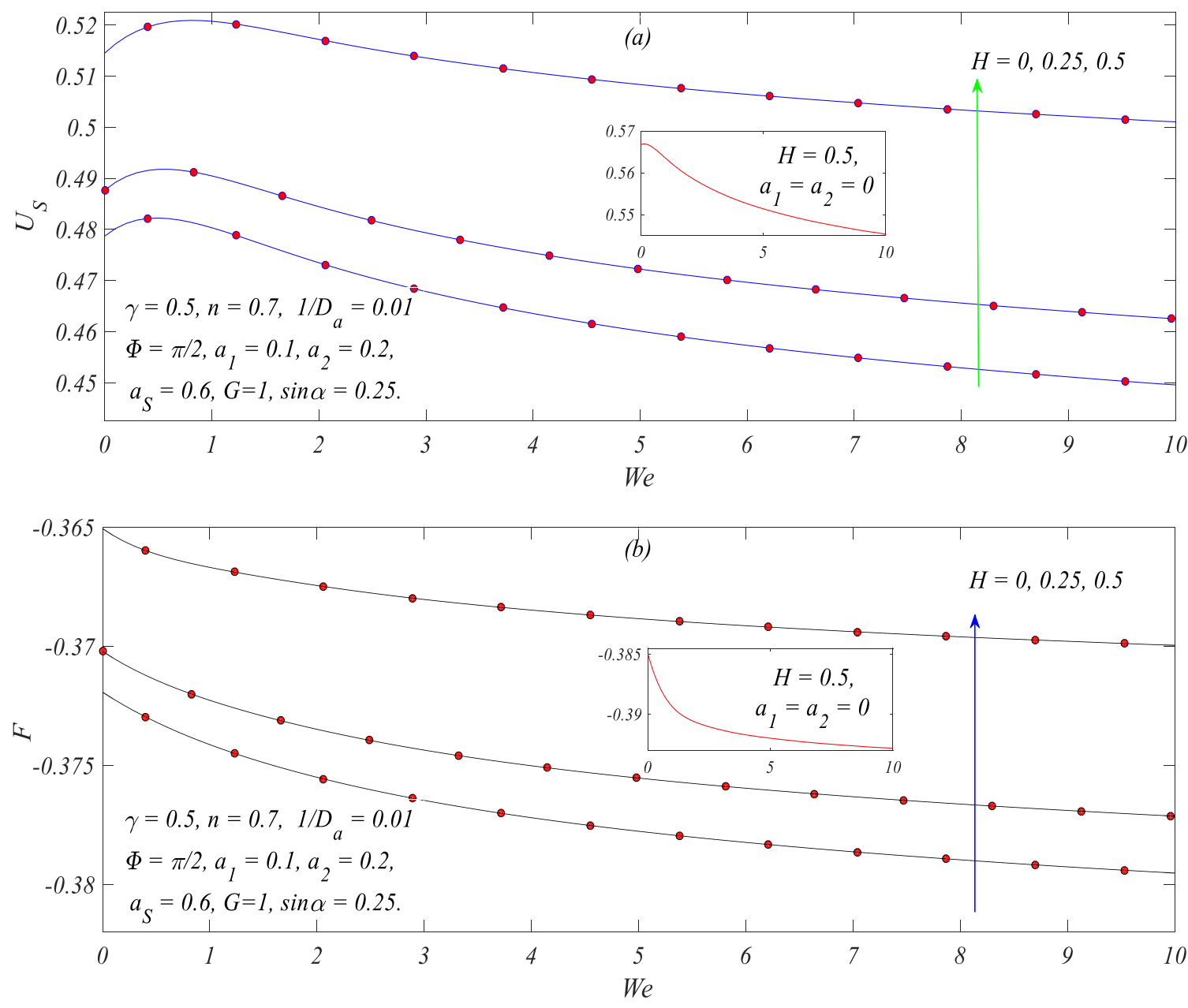


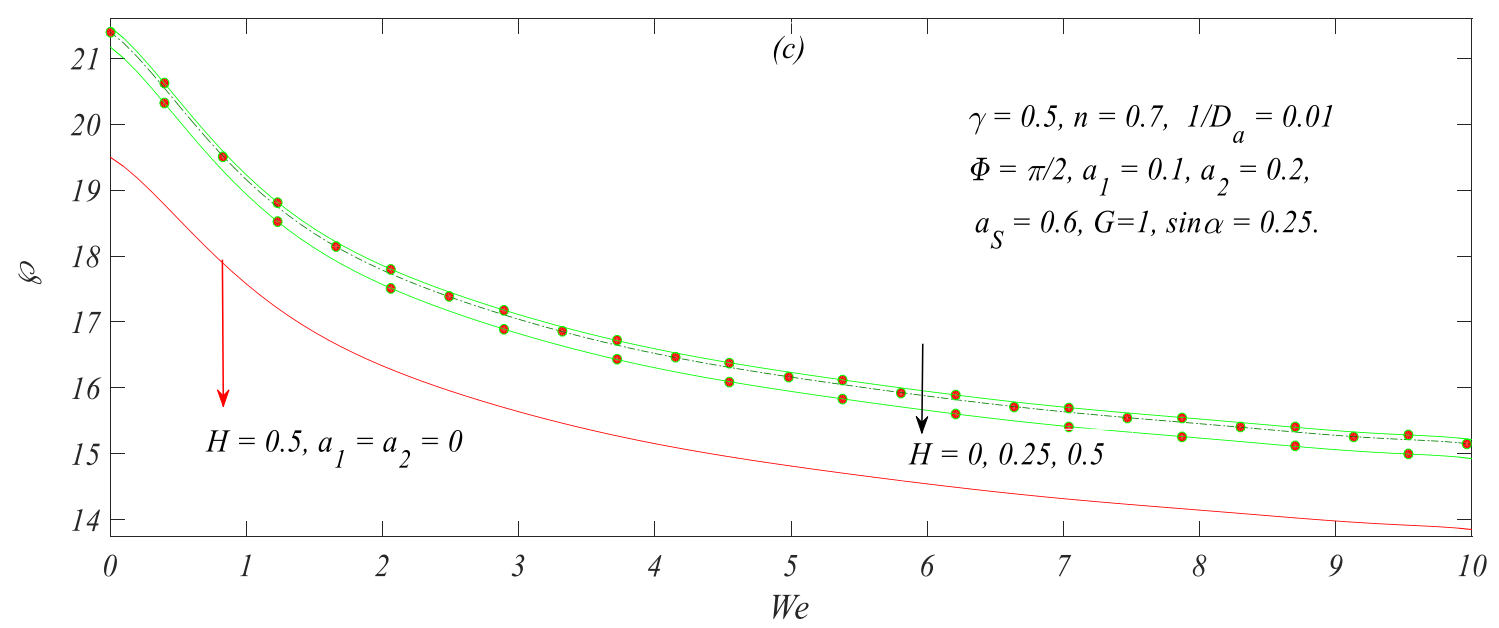

Fig.2. Variation of $(\boldsymbol{a})$ propelling speed $(\boldsymbol{b})$ flow rate and $(\boldsymbol{c})$ power losses against Weissenberg number and three different values of Hartmann number in active and passive channel.

It shows three outcomes: (i) when organism gets slower with varying fluid rheology it consumes less power (ii) Since MHD assist the organism to propel faster so the organism consumes less power in presence of magnetic field (iii) A wider rigid channel also provides an easy pathway to the swimmer which means swimmer needs more power to swim through a complex wavy active channel as compared to the passive one. Fig. 2 is plotted only for the shear-thinning mucus meanwhile the upcoming figure (Fig. 3) supports the fact that the shear-thickening mucus is exactly an opposite case of shear-thinning mucus.

Fig. 3 is plotted to investigate the behavior of propelling speed, flow rate and power expended as a function of power law index (n). Effects to porous medium on the swimming motion of the spermatozoa is also expounded. Figs. 3 (a), (b) and (c) are plotted for organism speed, flow rate and energy dissipation, respectively. One can witness from Fig. 3 that porous medium reduces the swimming speed, enhances the magnitude of flow rate and have negligible effects on power required by the swimmer. For comparatively larger values of Weissenberg number shearthickening propertise are more suitable to enhace the swimming speed (as shown in Fig. 3 (a)), while for small values of Weissenberg number $(W e<2)$ shear-thinnning propertise appear to be assistive. From Figs. 3 (b) and (c) it is clear that the magnitude of flow rate decreases and power increases when fluid rheology changes from shear-thinning to shear-thickening. Moreover, phase difference also effects organism swimming. When there is no phase difference between channel 
and organism wave the swimming speed and power dissipation reduces however flow rate enhances.
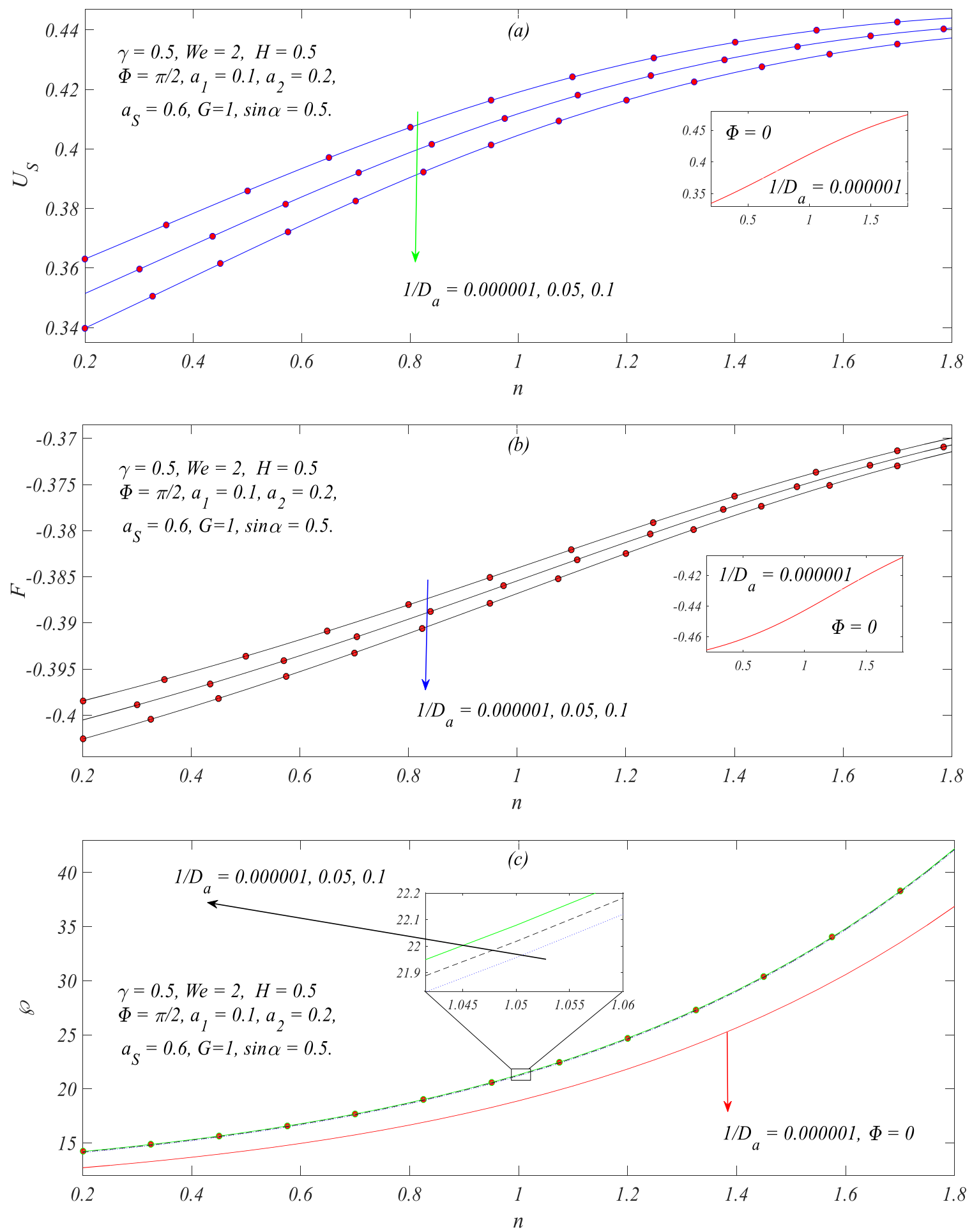

Fig.3. Variation of $(\boldsymbol{a})$ propelling speed $(\boldsymbol{b})$ flow rate and $(\boldsymbol{c})$ power losses against power law index and three 
different values of Darcy number in phase and out of phase scenario.

\subsection{ENERGY DISSIPATED IN DIFFERENT PHYSICAL SITUTAIONS}

In Figs. 2(c) and 3(c) the power is plotted after inserting different pairs of $F$ and $U_{S}$. However, if wave amplitude in the organism surface is tuned in such a way that it will maintain the same speed in two different situations the comparison of power will be more appropriate.

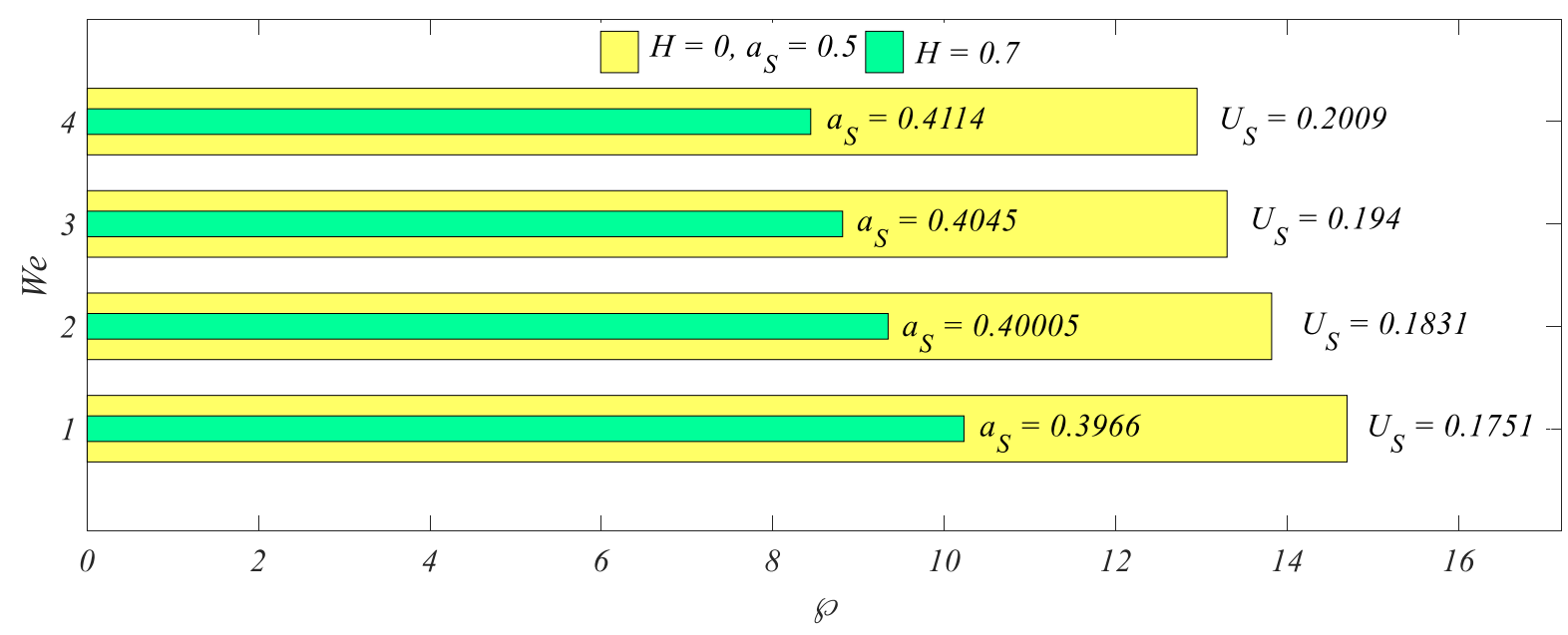

Fig. 4. Power dissipation in two different conditions i.e. with and without MHD (when organism is maintaining the same swimming speed in both situations). With $a_{1}=0.1, a_{2}=0.2, n=0.75, \gamma=0.5, \Phi=\pi / 2, G=1, \alpha=\pi / 6$ and $1 / D_{a}=0.1$.

In the present work we have discussed three possibilities for power comparison: $(i)$ in absence $(H=0)$ and presence $(H \neq 0)$ of $M H D(i i)$ in passive $\left(a_{1}=a_{2}=0\right)$ and complex wavy active $\left(a_{1}=0.2, a_{2}=0.3\right)$ channel (iii) in absence $\left(1 / D_{a} \longrightarrow 0\right)$ and presence $\left(1 / D_{a}>0\right)$ of porous medium. These cases are displayed in the form of bar charts in Figs. 4-6.

The first case is shown in Fig. 4. This bar graph reveals that in order to maintain the same speed in magnetic and non-magnetic environment an organism needs more energy in absence of magnetic field. This is because of the fact that MHD is an assistive force to the organism movement. 
Since Fig. 2 (a) already established that fact that the presence of complex wave in channel walls reduces the swimming speed so here Fig. $\mathbf{5}$ is in excellent agreement to that fact. It can be witness that the organism expended greater power in complex wavy channel as compared to rigid channel.

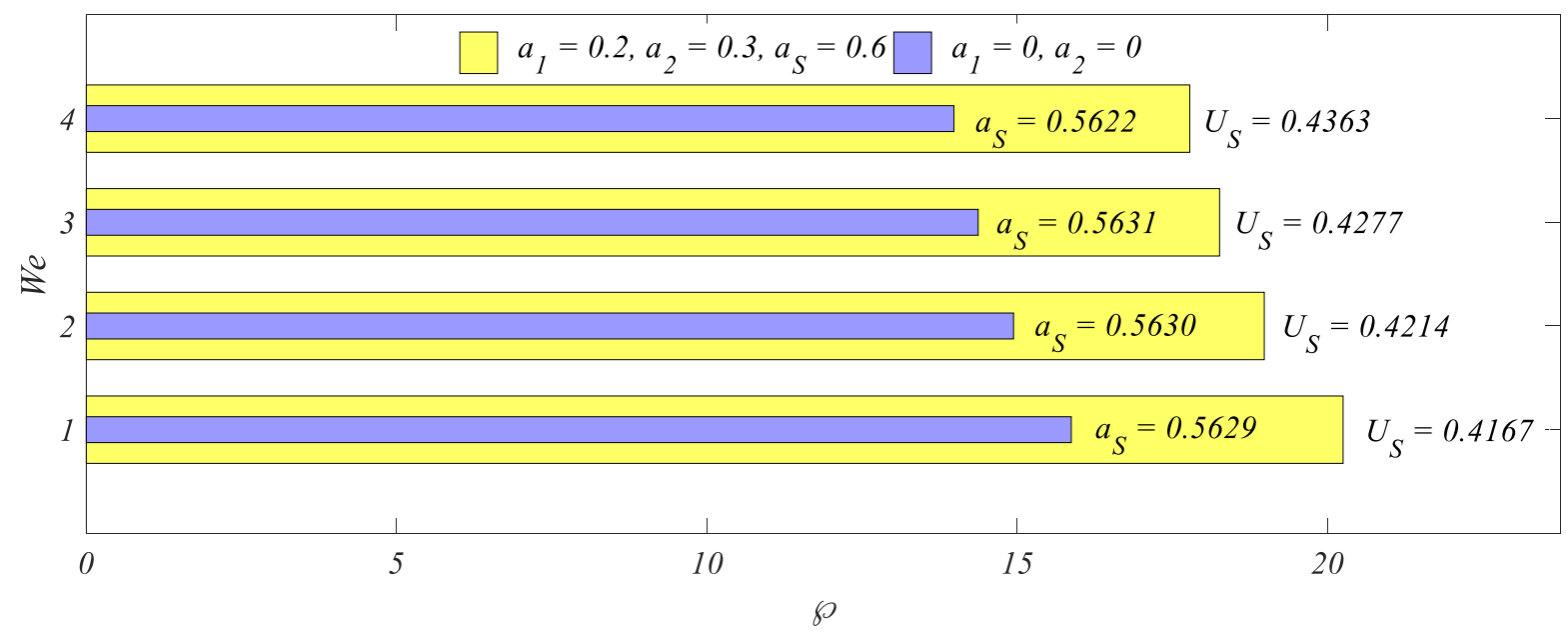

Fig.5. Power dissipation in two different conditions i.e. passive and complex wavy channel (when organism is maintaining the same swimming speed in both situations). With

$$
H=0.7, n=0.75, \gamma=0.5, \Phi=\pi / 2, G=1, \alpha=\pi / 6 \text { and } 1 / D_{a}=0.1 \text {. }
$$

Fig. 6 highlights the comparison of power expended by the organism propelling through porous and non-porous medium. It can be seen that the organism propelling through porous medium expended greater amount of energy as compared to non-porous medium.

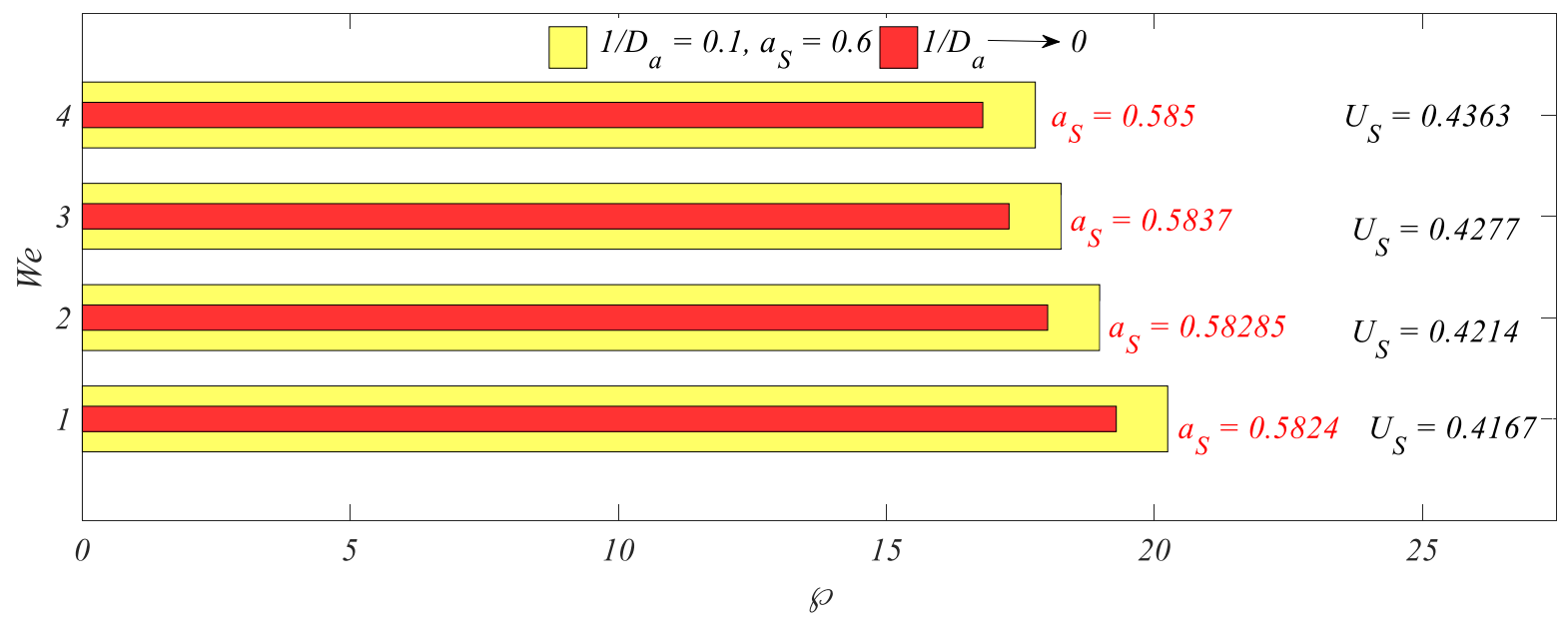


Fig. 6. Power dissipation in two different conditions i.e. with and without porous medium (when organism is maintaining the same swimming speed in both situations). With

$$
H=0.7, n=0.75, \gamma=0.5, \Phi=\pi / 2, G=1, \alpha=\pi / 6, a_{1}=0.2 \text { and } a_{2}=0.3 \text {. }
$$

\subsection{STREAMLINES IN BOTH PARTS OF THE CANAL}

The assessment of Carreau fluid's streamlines for various magnetic field strengths, phase difference, Darcy number, passive and complex wavy channel, fluid properties and swimming gait are presented in Figs. 7-9. In these figures an undulating surface of the micro-swimmer (in the central region) bounded between two complex wavy walls is displayed. A red arrow is indicating the direction of swimming spermatozoa.

Fig. 7 highlights the effects of magnetic field and phase difference on the streamline topologies. All the other parameters are fixed. When the organism is out of phase with the channel walls it propel faster and the surrounding fluid gets trapped. For $\Phi=0$ and $H=0$, there is no circulating zone and the level curves are similar in both regions (as shown in Fig. 7 (a)) while the streamlines for $\Phi=\pi / 4$ and $H=0$, exhibit a diverse trend in upper and lower side of the organism (as shown in Fig. 7 (b)). In comparison to Fig. 7 (a) (without MHD and no phase difference), Fig. 7 (c) is plotted for a considerable value of magnetic force and we can observe that a circulating zones also appear in the void regions. Comparing Fig. 7 (d) to Fig. 7 (b) $($ for $\Phi=\pi / 4)$ reveals that the strength of the circulating zones reduces with MHD effects.
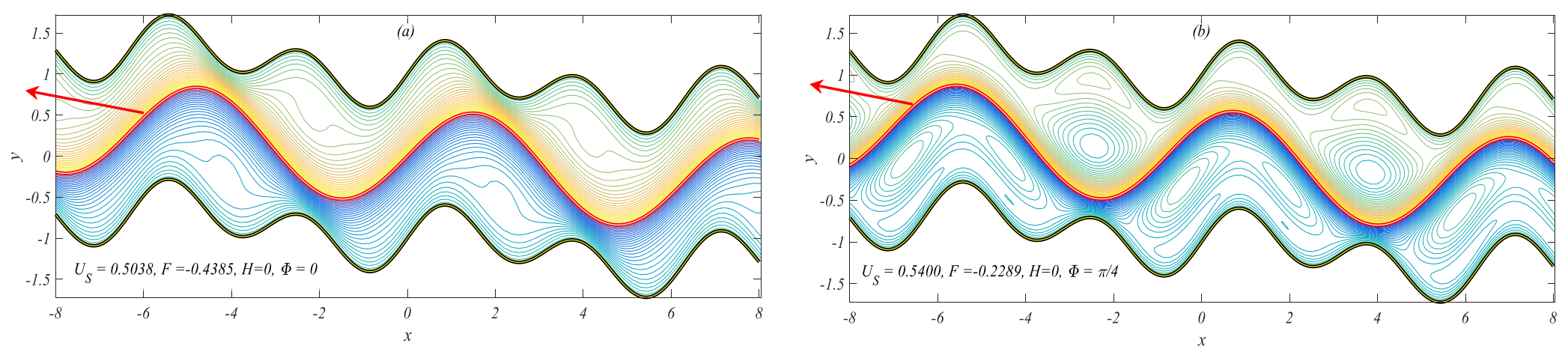

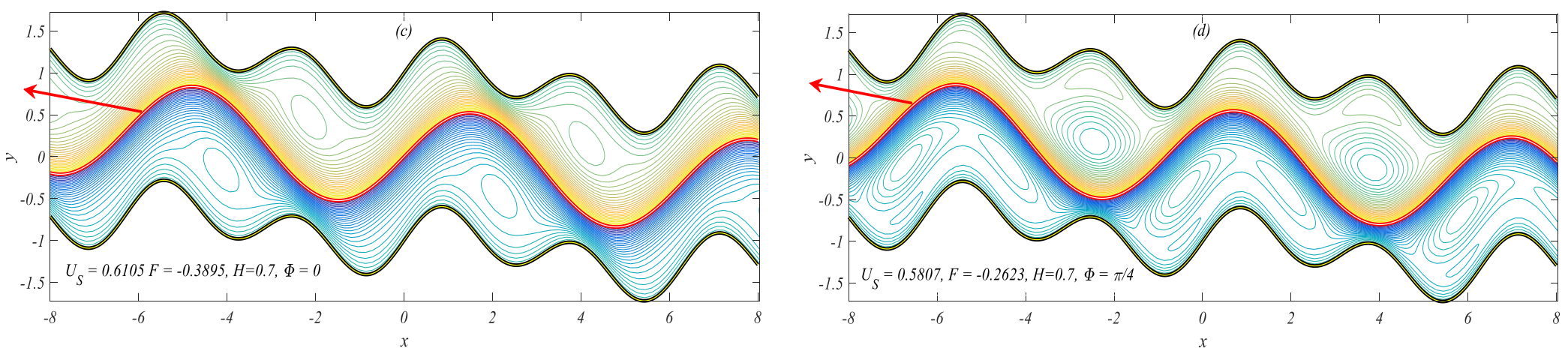

Fig. 7. Flow lines within a complex wavy inclined channel with four pairs of $U_{S}, F, H$ and $\Phi$ with $a_{S}=0.6, a_{1}=0.2, a_{2}=0.3, W e=5, n=1.3, \gamma=0.5$ and $1 / D_{a}=0.1$.

The eccentric behavior of stream function in rigid and complex wavy canal with/without porous medium effects are described in Fig. 8. Fig. 8 (a) is plotted to explore the porous effects within complex wavy canal while the same physical situation is displayed in Fig. 8 (b) for rigid canal
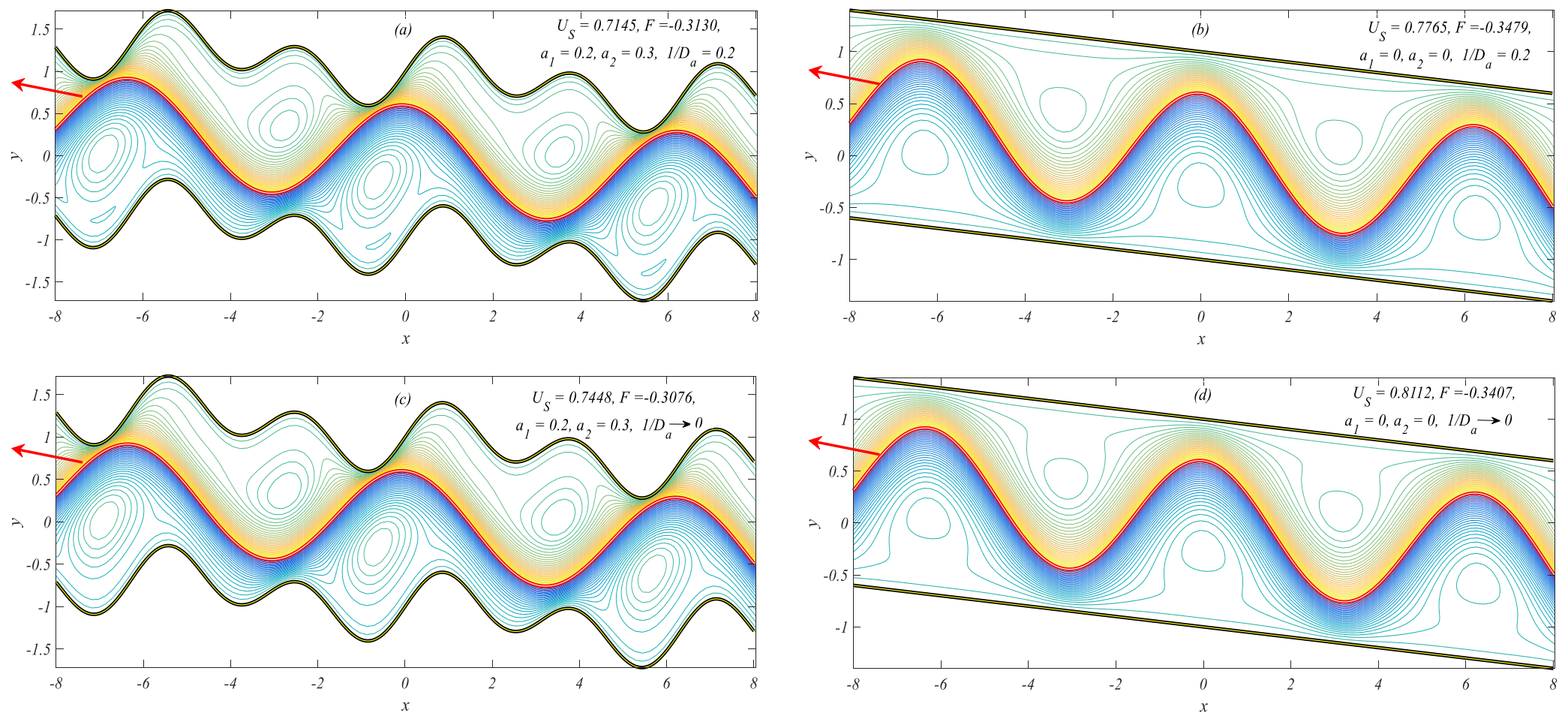

Fig. 8. Flow lines within a rigid and complex wavy inclined channel with four pairs of $U_{S}, F, a_{1}, a_{2}$ and $1 / D_{a}$ with $a_{S}=0.6, H=1$, We $=1, n=0.5, \gamma=0.5$ and $\Phi=\pi / 2$. 
It can be observed that the passive channel offers a less resistive pathway and the amount of trapped fluid reduces. In other words fluid flow is quite smooth in rigid channel as compared to complex wavy domain. On the other hand Figs. 8 (c) and (d) are plotted for negligible porous effects. By comparing these plots with plots. 8 (a) and (b) we arrive at the conclusion that porous effects enhances bolus size. In plots. 8 (c) small eddies (as shown in plots. 8 (a)) near the lower wall of the channel disappear.

Figs. 9(a)-(d) are shown for two different wave amplitudes in the organism surface and Weissenberg number. It can be seen from these plots that greater undulations results in formation of circulating zones, while in case of shear-thinning fluid high Weissenberg number enhaces the bolus size.
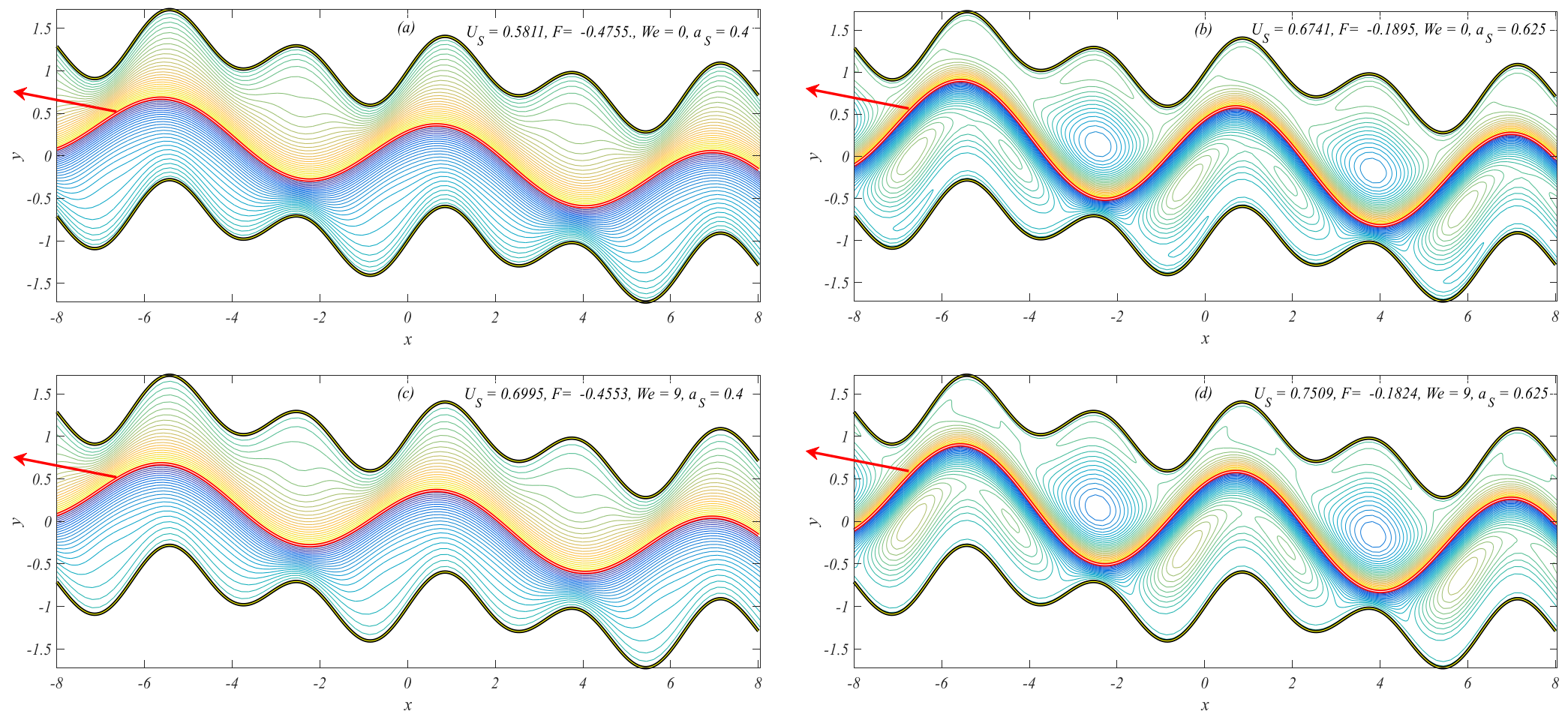

Fig.9. Flow lines within a complex wavy inclined channel with four pairs of $U_{S}, F$, We and $a_{S}$ with $n=0.5, a_{1}=0.2, a_{2}=0.3, H=1, \gamma=0.5, \Phi=\pi / 4$ and $1 / D_{a}=0.05$. 


\section{KEY FINDINGS}

The magnetic swimming problem at low Reynolds number with porous medium effects is explored. The tail of the micro-swimmer is approximated as two-dimensional sheet which is bounded in an inclined channel with complex wavy walls. The fluid around the swimming sheet is assumed to be Carreau fluid. The reduced boundary value problem is solved numerically by MATLAB routine bvp-4c. Newton Raphson algorithm is used to compute the flow rate and cell speed. The whole numerical procedure is tested by FDM. The computed results of organism speed, flow rate and power dissipation are plotted for various emerging parameters. The present work is concluded as follows:

- The organism propel slower through porous medium as compared to non-porous medium.

- The magnetic force applied in the downward direction enhances the swimming speed and reduce the power dissipation.

- The organism propel faster through passive channel with less energy losses as compared to complex wavy channel.

- The phase difference between channel and organism wave leads to faster propulsion.

- The fluid rheology plays an effective role in swimming motion. For small values of Weissenberg number shear-thinning rheology is suitable while for large Weissenberg number shear-thickening rheology is more appropriate.

- When the porous medium is not considered, channel is assumed to be passive and MHD is applied micro-organism can easily obtain that same speed (obtained in opposite situations) with less amount of work done.

- Greater undulations, phase difference and complex wavy nature creates circulating zones around the micro-swimmer.

\section{REFERENCES}

[1] G. I. Taylor, Analysis of the swimming of microscopic organisms. Proc. R. Soc. Lond. A, 209 (1951) 447-461. 
[2] G. I. Taylor, The action of waving cylindrical tails in propelling microscopic organisms. Proc. R. Soc. Lond. A, 211 (1952) 225-239.

[3] G. J. Hancock, The self-propulsion of microscopic organisms through liquids. Proc. $R$. Soc. Ser., A217 (1953) 96-121.

[4] J. Gray and G.J. Hancock, The propulsion of sea-urchin spermatozoa. J. Exp. Biol., 32 (1955) $802-814$.

[5] J. E. Drummond, Propulsion by oscillating sheets and tubes in a viscous fluid. J. Fluid Mech., 25 (1966) 787-793.

[6] A. J. Reynolds, The swimming of minute organisms. J. Fluid Mech., 23 (1965) 241-260.

[7] E. O. Tuck, A note on swimming problem. J. Fluid Mech., 31 (1968) 305-308.

[8] W. J. Shack and T. J. Lardner, A long wavelength solution for a microorganism swimming in a channel. Bull. Math. Biol., 36 (1974) 435-444.

[9] J. B. Shukla, B. R. P. Rao and R. S. Parihar, Swimming of spermatozoa in cervix: effects of dynamical interaction and peripheral layer viscosity. J. Biomech., 11, (1978) 15-19.

[10] R. E. Smelser, W. J. Shack and T. J. Lardner, The swimming of spermatozoa in an active channel. J. Biomech., 7 (1974) 349-355.

[11] J. B. Shukla, P. Chandra and R. Sharma, Effects of peristaltic and longitudinal wave motion of the channel wall of movement of micro-organisms: application to spermatozoa transport. J. Biomech., 21 (1988) 947-954.

[12] G. Radhakrishnamacharya and R. Sharma, Motion of a self-propelling micro-organism in a channel under peristalsis: effects of viscosity variation. Nonlinear Anal. Model., 12 (2007) 409-418.

[13] T. K. Chaudhury, On swimming in a viscoelastic liquid. J. Fluid Mech., 95 (1979) 189197. 
[14] M. Sajid, N. Ali, O. Anwar Bég, A.M. Siddiqui, Swimming of a singly flagellated microorganism in a magnetohydrodynamic second order fluid. J. Mech. Med. Biol., 17 (2017) 1750009.

[15] L.D. Sturges, Motion induced by a waving plate, J. Non Newton Fluid Mech., 8 (1981) 357-364.

[16] E. Lauga, Propulsion in a viscoelastic fluid. Phys. Fluids, 19 (2007) 083104.

[17] M. Sajid, N. Ali, A.M. Siddiqui, Z. Abbas and T. Javed, Effects of permeability on swimming of a micro-organism in an Oldroyd-B Fluid. J Porous Media, 17 (2014) 59-66.

[18] N. Ali, M. Sajid, Z. Abbas and O. Anwar Bég, Swimming dynamics of a micro-organism in a couple stress fluid: a rheological model of embryological hydrodynamic propulsion. $J$. Mech. Med. Biol., 17 (2017) 1750054.

[19] P. Sinha, C. Singh and K.R. Prasad, A microcontinuum analysis of the self-propulsion of the spermatozoa in the cervical canal, Int .J. Engng. Sci., 20 (1982) 1037-1048.

[20] D. Philip and P. Chandra, Self-propulsion of spermatozoa in microcontinua: effects of transverse wave motion of channel walls. Arch. Appl. Mech., 66 (1995) 90-99.

[21] N. J. Balmforth, D. Coombs and S. Pachmann, Microelastohydrodynamics of swimming organisms near solid boundaries in complex fluids. Q. J. Mech. Appl. Math., 63 (2010) 267-294.

[22] Thomas R. Ives, and Alexander Morozov, The mechanism of propulsion of a model microswimmer in a viscoelastic fluid next to a solid boundary, Phys. Fluids, 29 (2017) 121612.

[23] S. Sudo, S. Segawa and T. Honda, Magnetic swimming mechanism in a viscous liquid, $J$. Intell. Mater Syst. Struct. 17 (2006) 729-736.

[24] F.Z. Temel and S. Yesilvurt, Confined swimming of bio-inspired magnetic microswimmers in rectangular channels, 67th Annual Meeting of the APS (American Physical Society) Division of Fluid Dynamics, San Francisco, California: APS November, 2014. 
[25] F.Z. Temel, A.E. Bezer and S. Yesilvurt, Navigation of mini-swimmers in channel networks with magnetic fields, IEEE (Institute of Electrical and Electronics Engineers), Int Conf Robotics and Automation (ICRA 2013), USA: IEEE May, 2013.

[26] D.R. Bond, Electrode-reducing microorganisms that harvest energy from marine sediments, Science 295 (2002) 483-485.

[27] D.R. Lovely, The microbe electric: Conversion of organic matter to electricity, Curr Opin Biotechnol., 19 (2008) 564-571.

[28] A.R. Ansari, A.M. Siddiqui and T. Hayat, An analysis of the swimming problem of a singly flagellated micro-organism in an MHD fluid, Nonlinear Dyn 51 (2008) 477-481.

[29] H. Gadelha, On the optimal shape of magnetic swimmers, Regul Chaotic Dyn 18 (2013) $75-84$.

[30] M. Belovs and A. Cebers, Ferromagnetic microswimmer, Phys Rev E, 79 (2009) 051503.

[31] W. Gao, D. Kagan, O. S. Pak, C.Clawson, S. Campuzano, E. Chuluun-Erdene, E. Shipton, E. E. Fullerton, L. Zhang, E. Lauga and J. Wang, Cargo-towing fuel-free magnetic nanoswimmers for targeted drug delivery, Small 8 (2012) 460-467.

[32] E. Gauger, M. Downton and H. Stark, Fluid transport at low Reynolds number with magnetically actuated artificial cilia, Eur Phys J. E, 28 (2009) 231-242.

[33] Z. Asghar, N. Ali, M. Sajid and O. Anwar Bég, Magnetic microswimmers propelling through biorheological liquid bounded within an active channel. J. Magn. Magn. Mater., 486 (2019) 165283.

[34] A.R. Ansari, A.M. Siddiqui and T. Hayat, An analysis of the swimming problem of a singly flagellated micro-organism in an MHD fluid flowing through a porous medium, Nonlinear Anal Real World Appl 11 (2010) 22-28.

[35] A.A. Farooq, D. Tripathi, and T. Elnaqeeb, On the propulsion of micropolar fluid inside a channel due to ciliary induced metachronal wave. Appl Math Comput., 347 (2019) 225235. 
[36] N.S. Akbar, D. Tripathi, Z. H. Khan and O. Anwar Bég, Mathematical modelling of pressure-driven micropolar biological flow due to metachronal wave propulsion of beating cilia. Math. Biosci., 301 (2018) 121-128.

[37] K. Ramesh, D. Tripathi, and O. Anwar Bég, Cilia-assisted hydromagnetic pumping of biorheological couple stress fluids. J. Propul. Power Research (2019).

[38] N.S. Akbar, M. Shoaib, D. Tripathi, S. Bhushan, and O. Anwar Bég, Analytical approach to entropy generation and heat transfer in CNT-nanofluid dynamics through a ciliated porous medium. J. Hydrodyn., 30(2) (2018) 296-306.

[39] N.S. Akbar, D. Tripathi, Z. H. Khan and O. Anwar Bég, Mathematical model for ciliaryinduced transport in MHD flow of $\mathrm{Cu}-\mathrm{H}_{2} \mathrm{O}$ nanofluids with magnetic induction. Chin. J. Phys., 55(3) (2017) 947-962.

[40] N.S. Akbar, A.W. Butt, D. Tripathi, and O. Anwar Bég, Physical hydrodynamic propulsion model study on creeping viscous flow through a ciliated porous tube. Pramana $88(3)$ (2017) 52 .

[41] N.S. Akbar, D. Tripathi, O. Anwar Bég and Z. H. Khan, MHD dissipative flow and heat transfer of Casson fluids due to metachronal wave propulsion of beating cilia with thermal and velocity slip effects under an oblique magnetic field. Acta Astronaut., 128 (2016) 112.

[42] F. A. Morrison, Understanding Rheology, Oxford University Press Inc. New York, (2001).

[43] J. R.Vélez-Cordero, Eric Lauga, Waving transport and propulsion in a generalized Newtonian fluid. J. Non-Newtonian Fluid Mech., 199 (2013) 37-50.

[44] N. Ali, Z. Asghar, O. Anwar Bég and M. Sajid, Bacterial gliding fluid dynamics on a layer of non-Newtonian slime: Perturbation and numerical study. J. Theoretical Biology, 397 (2016) 22-32.

[45] Z. Asghar, N. Ali, and M. Sajid, Analytical and numerical study of creeping flow generated by active spermatozoa bounded within a declined passive tract. Eur. Phys. J. Plus, 134 (2019) 9 
[46] T. Hayat, S. Farooq, A. Alsaedi and B. Ahmad, Hall and radial magnetic field effects on radiative peristaltic flow of Carreau-Yasuda fluid in a channel with convective heat and mass transfer. J. Magn. Magn. Mater., 412 (2016) 207-216. 